\title{
Effects on endometrial mechanical stimulation (healing) in normal intrauterine insemination cycles
}

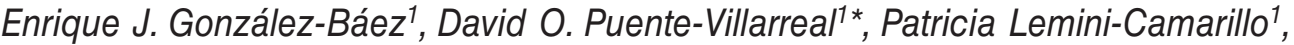 \\ Geraldina Guerrero-González², José G. Garza-Leal², and Donato Saldívar-Rodríguez² \\ ${ }^{1}$ Reproduction Biology Service and Center for Reproductive Medicine (CeUMER acronym in Spanish); ${ }^{2}$ Department of Obstetrics and Gynecology, \\ "Dr. José Eleuterio González" University Hospital, Universidad Autónoma de Nuevo León", Monterrey, Nuevo Leon, Mexico
}

\begin{abstract}
Objective: The objective of the study was to investigate the effect of healing on the results of intrauterine insemination cycles. Healing stimulation consists of placing a pipelle insemination cannula vaginally through the cervix, until it touches the uterine fundus and remove it. Material and methods: An experimental, longitudinal, prospective, comparative, and non-blinded study was performed with patients from the "Dr. José Eleuterio González," University Hospital, who attended the Center for Reproductive Medicine (CeUMER) who underwent controlled ovarian stimulation and intrauterine insemination. Two groups were formed: a control group consisting of 40 cycles and a study group of 40 cycles, in which healing was performed on the day of the application of choriogonadotropin alfa as the trigger for ovulation. Results: A clinical pregnancy rate of $17.5 \%$ and biochemical pregnancy rate of 5\% were found in the endometrial mechanical stimulation study group undergoing intrauterine inseminations, while the control group obtained a clinical pregnancy rate of $20 \%$. They did not show biochemical pregnancies in the control group with a biochemical pregnancy rate without having a statistical significance $(p=0.15)$. There was no significant impact on clinical pregnancy when performing healing. Conclusions: There was no significant impact on the clinical pregnancy rate in the study group when healing was performed. The presence of biochemical pregnancy was evidenced only in the group with stimulation, but no significant value was presented. The other variables that were evaluated during the study did not have a significant difference between the two groups, nor in the association with clinical and biochemical pregnancy.
\end{abstract}

Key words: Endometrial stimulation. Fertility. Intrauterine insemination. Healing.

\section{Introduction}

Infertility is a common problem, affecting one in six couples. It can be defined as the inability to complete a pregnancy after a year of sexual relations without any contraceptive measures or treatment ${ }^{1}$.

Despite scientific breakthroughs in reproductive medicine, there is no proof that the process of embryo implantation can be controlled by a specialist in human reproduction. If it were a controlled process, pregnancy rates would increase significantly among infertile couples who undergo both high and low complexity treatments ${ }^{1}$.

Today, two main techniques are considered as the treatment for infertile couples, intrauterine insemination, and in vitro fertilization (IVF), also considered low and high complexity treatments, respectively².

\section{Correspondence:}

${ }^{*}$ David O. Puente-Villarreal

E-mail: davidpuente1287@ @otmail.com
Available online: 17-03-2020

Date of reception: 20-12-2018

Date of acceptance: 13-11-2019 DOI: 10.24875/RMU. 19000045
Medicina Universitaria. 2020;22(1):21-26 www.medicinauniversitaria.org

(1) CC BY-NC-ND license (http://creativecommons.org/licenses/by-nc-nd/4.0/). 
When performing a low complexity procedure, such as intrauterine insemination, a controlled ovarian stimulation is carried out beforehand, using menotropins, which during the follicular phase of the menstrual cycle will help the recruitment and growth of dominant follicles. Once these follicles reach a size $>16 \mathrm{~mm}$, they are considered qualified to perform intrauterine insemination ${ }^{3}$. The reported success rate of intrauterine insemination is approximately $15-20 \%$ per cycle. After four tries at intrauterine insemination, an accumulated rate of $45-50 \%$ can be achieved ${ }^{3}$. The result of a successful embryo implantation process is the serum increase of the human chorionic gonadotropin hormone, after 2 weeks of intrauterine insemination procedure ${ }^{2}$.

In 2007, in a study conducted in Iran, they were able to observe that if a mechanical injury was caused in the endometrium, this would undergo an inflammatory process, followed by a chain-secretion of cytokines, prostaglandins, chemotactic factors, and growth factors that later induced a repair process of the endometrial tissue, called "Healing endometrial"4.

It has been previously documented that the effects of endometrial mechanical stimulation trigger the genetic expression of necessary factors for implantation, such as laminin alpha-4, integrin alpha-6, metalloproteinase, and glycodelin $\mathrm{A}$, which take part in endometrial repair. There is also a reduction of endometrial thickness, which can occur due to the physiological secretion of estrogens as a result of controlled ovarian stimulation in assisted reproduction techniques ${ }^{5}$.

This repaired tissue promotes the attachment of the embryo and the endometrium, thus increasing implantation rate and, consequently, pregnancy rate ${ }^{4}$.

Embryo implantation is a 3 -stage process (apposition, adhesion, and invasion), involving a synchronized cross-talk between a receptive endometrium and a functional blastocyst. This ovarian-steroid-dependent phenomenon can only take place during the implantation window, a self-limited period of endometrial receptiveness, which extends between the $20^{\text {th }}$ and $24^{\text {th }}$ days of the menstrual cycle $^{6}$.

Finally, when the cells attach to the endometrium in their entirety, a fusion called syncytiotrophoblast is created, which will eventually be the origin of the placenta. The syncytiotrophoblast will be the place of the secretion of the chorionic gonadotropin hormone, being the moment when the serum can be detected. Moreover, it will be in charge of avoiding the involution of the corpus luteum until full formation of the placenta takes place ${ }^{6}$.

In our Reproductive Center at the "Dr. Jose Eleuterio Gonzalez" University Hospital, infertility problems are treated according to the degree of pathology that the patients to be studied present, since there is a low-complexity department as well as a high-complexity one.

When patients present criteria and characteristics suitable for a low-complexity procedure, intrauterine insemination with controlled ovarian stimulation is the first treatment option, sending the non-candidate patients to the high-complexity department, where they will undergo IVF procedures.

The endometrial mechanical stimulation procedure has reports of ambiguous studies, predominating encouraging results, with a better implantation rate ${ }^{4}$.

This study was conducted in view of the need and desire to increase clinical pregnancy rates, searching to clarify the factors that may be linked to the results of clinical pregnancy.

\section{Materials and methods}

The present study was performed at the Reproductive Medicine Center of the School of Medicine and "Dr. Jose E. Gonzalez" University Hospital of the Autonomous University of Nuevo Leon (UANL by its Spanish acronym), including patients who attended the center between March 2014 and 2015 with an infertility diagnosis and who underwent controlled ovarian stimulation and intrauterine insemination, with an age range of 2039 years old. Patients who had over four failed cycles of artificial insemination were excluded from the study.

The study included 80 patients, who were selected following the protocols for candidates for artificial insemination at our center, and two groups were formed; Group A and Group B. Each group included 40 patients who underwent intrauterine insemination cycles. Group A was named "control," while Group B was named "cases," where patients received treatment with mechanical stimulation of de endometrium (healing) the day of the application of choriogonadotropin alfa.

Healing stimulation consisted of placing a pipelle (3.1 $\mathrm{mm}$ thick) insemination cannula vaginally through the cervix, until it touches the uterine fundus and removing it, performed the day of the follicle injection, with $250 \mathrm{UI}$ of choriogonadotropin alfa subcutaneously.

The insemination was conducted by placing the patient in the lithotomy position, a vaginal speculum was set, cervical mucus was cleaned using a swab, a catheter was introduced for a Sidney-type insemination up to the interior of the uterine cavity, $0.4 \mathrm{ml}$ of previously qualified semen were deposited, and finally the patient was allowed to rest for 15 min subsequent to the procedure. 
Any patient who showed positive results in the immunological pregnancy test (IPT) was considered as an accomplished biochemical pregnancy and a patient with a positive IPT where the presence of an embryo with a fetal heart rate using a transvaginal ultrasound was considered a clinical pregnancy. We used an ultrasound with General Electric Healthcare Austria Co. model VOLUSON E8, serial number E32129-2016-03, property of the Department of Obstetrics of the Gynecology and Obstetrics Service at the "Dr. Jose E. Gonzalez" University Hospital of the Autonomous University of Nuevo Leon.

\section{Ethical considerations}

The present study was approved by the Ethics Committee and the Research Committee at the School of Medicine and the "Dr. Jose E. Gonzalez" University Hospital of the Autonomous University of Nuevo Leon with the registration number Gl16-00004. Moreover, the study was conducted under a signed informed consent by the patients previous to conducting the procedure. The team of researchers states not to have a conflict of interest in the making of this study.

\section{Statistical analysis}

Statistical analysis was performed using the Epi Info 7 software for Windows version 20.0 and Microsoft Excel version 2010. The data were described as range, median, and standard deviation for numerical parametric, with frequency and ratio for categorical variables. The difference between two independent groups was analyzed through the Student's t-test for numerical values and $\chi^{2}$ for polyatomic categorical variables, as well as an odds ratio (OR) to determine the link of risks with a confidence interval $(\mathrm{Cl})$ of $95 \%$.

\section{Results}

A total of 80 cycles were integrated, divided into two groups with paired-sample tests, subsequent to the gathering of information, and performing the corresponding statistical analysis.

The average age of the patients who presented a clinical pregnancy was $32(p=0.57)$, with an average body mass index (BMI) of $27 \mathrm{~kg} / \mathrm{m}^{2}(p=0.9)$, the number of menotropin doses was $16(p=0.48)$, and the number of dominant follicles was $2.2(p=0.75)$. Follicle size was 18.56 millimeters $(p=0.29)$, the size of the endometrium was 9.87 millimeters $(p=0.91)$, registered spermatozoid
Table 1. Association of clinical pregnancy cases

\begin{tabular}{|l|c|c|c|c|c|}
\hline Variable & Average & SD & Min & Max & p \\
\hline Age & 32.64 & 3.44 & 22 & 39 & 0.57 \\
\hline $\begin{array}{l}\text { Body mass index } \\
\text { (kg/m²) }\end{array}$ & 27.27 & 5.05 & 20 & 45 & 0.9 \\
\hline Merapur ampules & 16.8 & 6.95 & 6 & 35 & 0.48 \\
\hline Number of follicles & 2.29 & 1.2 & 1 & 5 & 0.75 \\
\hline $\begin{array}{l}\text { Endometrial } \\
\text { size (mm) }\end{array}$ & 9.87 & 1.82 & 7 & 16 & 0.91 \\
\hline Sperm motility & 73.33 & 23.52 & 47 & 133 & 0.06 \\
\hline $\begin{array}{l}\text { Progressive } \\
\text { mobiles M }\end{array}$ & 29.35 & 20.75 & 3 & 110 & 0.2 \\
\hline $\begin{array}{l}\text { Number of cycles } \\
\text { Sperm }\end{array}$ & 1.35 & 0.59 & 1 & 3 & 0.34 \\
\hline $\begin{array}{l}\text { concentration } \\
\text { (total millions) }\end{array}$ & 53.3 & 32.6 & 4 & 178 & 0.12 \\
\hline \begin{tabular}{l} 
Follicle size (mm) \\
\hline
\end{tabular} & 18.56 & 1.26 & 16 & 22 & 0.29 \\
\hline
\end{tabular}

The values presented were compared with a Student's t-test.

Table 2. Risk association between healing groups

\begin{tabular}{|l|c|c|c|c|c|}
\hline \multirow{2}{*}{ Variable } & \multirow{2}{*}{ Frequency } & \multicolumn{3}{|c|}{ Odds ratio Cl 95\% } & \multirow{2}{*}{ p } \\
\cline { 2 - 5 } & & RM & Min & Max & \\
\hline Clinical pregnancy & 7 & 0.84 & 0.27 & 2.61 & 0.77 \\
\hline $\begin{array}{l}\text { Biochemical } \\
\text { pregnancy }\end{array}$ & 2 & SD & SD & SD & 0.15 \\
\hline Primary infertility & 29 & 1.26 & 0.48 & 3.31 & 0.62 \\
\hline
\end{tabular}

The values presented were compared using the $\chi^{2}$ test. Cl: confidence interval; OR: odds ratio.

motility was $73 \%(p=0.06)$, the concentration of semen had an average of 53 million ( $p=0.12$ ), and the number of cycles to which the patients were subject to was 1.3 events $(p=0.34)$, these values were not considered statistically significant (Table 1).

We observed a clinical pregnancy rate of $17.3 \%$ in the treatment group and $20 \%$ in the control group, not observing a statistically significant difference $(p=0.77)$. In the treatment group, a rate of biochemical pregnancies of $5 \%$ was documented, while the control group was $0 \%$, not observing a statistically significant difference $(p=0.15)$.

Likewise, we proceeded to the determination of linked risks through the estimation of the OR where observations showed that clinical pregnancy linked to 
Table 3. Distribution of the treatment group against associated factors

\begin{tabular}{|l|c|c|c|c|c|c|c|}
\hline \multirow{2}{*}{ Variable } & \multicolumn{9}{|c|}{ Case groups } \\
\cline { 2 - 8 } & \multicolumn{3}{|c|}{ Healing } & \multicolumn{3}{|c|}{ No healing } \\
\cline { 2 - 8 } & Average & Min & Max & Average & Min & Max \\
\hline Age & 33.37 & 31 & 39 & 32.3 & 22 & 39 \\
\hline Body mass index (kg/m) & 27.87 & 20 & 34 & 26.64 & 20 & 45 & 0.15 \\
\hline Sperm concentration (total millions) & 49.74 & 4 & 146 & 62.27 & 6 & 178 & 0.08 \\
\hline Progressive mobiles M & 32.21 & 4.9 & 110 & 29.52 & 3 & 81 & 0.58 \\
\hline Sperm motility & 78.35 & 15 & 133 & 72.93 & 82.5 & 98 & 0.29 \\
\hline Endometrial size (mm) & 9.8 & 7 & 16 & 9.97 & 7 & 14 & 0.67 \\
\hline Follicle size (mm) & 18.65 & 16 & 22 & 18.62 & 16 & 22 & 0.91 \\
\hline Merapur ampules & 17.3 & 6 & 35 & 16.83 & 7 & 33 \\
\hline Number of follicles & 2.27 & 1 & 5 & 2.35 & 1 & 5 \\
\hline Number of cycles & 1.37 & 1 & 4 & 1.4 & 1 & 0.76 \\
\hline
\end{tabular}

The values presented were compared with a Student's t-test.

the treatment group obtained an $\mathrm{OR}=0.84(\mathrm{Cl}=95 \%$, $0.27-2.61$ ), biochemical pregnancy was documented at $5 \%$ among the treatment group, and no cases occurred in the control group. The type of primary infertility obtained was $\mathrm{OR}=1.26(\mathrm{Cl}=95 \%, 0.48-3.31)$. Hence, it is not possible to determine a link of risk since all values are considered indeterminate (Table 2).

Last but not least, through the Student's t-test, we were able to determine interdependency between variables versus the treatment group, where they did not obtain any results which were statistically significant (Table 3).

\section{Discussion}

In 2013, there was a similar study to the one at our center, at the Department of Gynecology and Obstetrics (AlIMS) in New Delhi. A case-control study was conducted, recruiting 72 patients who underwent controlled ovarian stimulation with intrauterine insemination with healing as the "case" group and 72 patients with controlled ovarian stimulation with intrauterine insemination without healing as the "control" group, reporting a clinical pregnancy rate of $31.9 \%$ in the case group and $16.7 \%$ in the control group, said difference between groups being statistically significant $(p=0.02)$.

These results contrast with those obtained in the present study since it presented a clinical pregnancy rate of
$17.3 \%$ in the case group versus $20 \%$ in the control group. This difference was not statistically significant $(p=0.77)$.

The clinical pregnancy rate in patients who underwent endometrial mechanical stimulation was lower than that reported by previous studies. This can be explained due to the different methodologies to produce Healing since, in the AllMS, endometrial mechanical stimulation was performed with a \#4 Karman cannula (4 mm thick), which has a greater thickness than the pipelle cannula used in our study. When performing the introduction of a cannula of greater thickness to the uterus, more significant damage will be caused, which will trigger a greater activation and concentration of pro-inflammatory factors, cytokines, and growth factors such as interleukin 11 and type insulin-like growth factor-1 growth factor, which in turn will cause a larger endometrial repair area with consequently a more suitable bedding for embryo implantation?

Barash et al. conducted a study where IVF treatments and embryo transferences were preceded by repeated endometrial biopsies, in a randomized group of 45 of 134 patients. The transference of a similar number of embryos $(3.4 \pm 1.0$ and $3.1 \pm 0.9$ in experimental and control patients, respectively) which led to implantation rates $(27.7 \%$ vs. $14.2 \%, p=0.00011)$, clinical pregnancy (66.7\% vs. $30.3 \%, p=0.00009)$, and live births by embryo transference $(48.9 \%$ vs. $22.5 \%$, $p$ $=0.016$ ), reported more than double the effectiveness in the experimental group compared to control groups ${ }^{8}$. 
Gnainsky et al. ${ }^{9}$ proved that the endometrial injury induces an inflammatory reaction characterized by the affluence of macrophages and dendritic cells, resulting in an increase in the local production of cytokines and growth factors, which in turn promote decidual proliferation. Moreover, the authors were able to establish a positive correlation between the concentration of macrophage inflammatory protein IB, the macrophages of the cytokines, and the establishment of pregnancies in patients who received an endometrial injury ${ }^{10}$.

The rate of clinical pregnancies improved by performing endometrial mechanical stimulation was not statistically significant. The rate of biochemical pregnancies did not present a significant impact in the group of patients with mechanical stimulation; however, there were no biochemical pregnancies in the control group, establishing better implantation when the endometrial mechanical stimulation was performed. The number of the cycle, follicle size, BMI, age, endometrial thickness, day of insemination, and seminal quality was factors that did not present a statistical impact on the clinical pregnancy rate.

Concerning the rest of the variables, statistically significant differences were found in the number of cycles, follicle size, BMI, age, endometrial thickness, day of insemination, and seminal quality between the treatment and control groups, both being very homogeneous, without presenting relevance regarding clinical pregnancy rates, which was the main objective of the study, and which had not been evaluated in previous studies.

Concerning the moment in which the endometrial injury is produced, it seems logical to perform the intervention in the proliferative or luteal phase of the menstrual cycle immediately preceding the ovarian stimulation cycle. In a study conducted by Zhou et al. and executed by Guven et al., the endometrial injury was performed approximately 2-3 weeks before the embryo transfer, representing the shortest period related to a positive effect of healing over endometrial receptiveness ${ }^{11,12}$.

The same thing occurred with the relationship between the variables to quantify patients who accomplished clinical pregnancy with those who did not, since no statistically significant difference was found.

These results were expected, since all patients complied with the criteria established to begin intrauterine insemination, of homogeneous characteristics.

Within the limitations of this study is the fact that the sample size considered was 80 patients included for the sample. It is possible that when raising the number of cases and controls undergoing endometrial healing, there is statistical significance for the accomplishment of clinical or biochemical pregnancy.

\section{Conclusions}

Several interventions to increase the success rates of IVF have been proposed. However, very few are directed at improving endometrial receptivity. Optimal synchronization and the time of performing the healing in relationship to the IVF cycle or artificial insemination are far from being resolved.

Our study did not show a link between performing endometrial healing and an increase in pregnancy rates.

Due to the fact that IVF success rate is modest, these results are of interest, since the proposed intervention is not costly nor requires much time, and apparently lacks significant complications.

Therefore, our study opens a window to perform new studies, which involve patients who undergo IVF and embryo transference with the aim of increasing the chance of pregnancy.

\section{Conflicts of interest}

The authors declare that they have no conflicts of interest.

\section{Ethical disclosures}

Protection of human and animal subjects. The authors declare that no experiments were performed on humans or animals for this study.

Confidentiality of data. The authors declare that they have followed the protocols of their work center on the publication of patient data.

Right to privacy and informed consent. The authors have obtained the written informed consent of the patients or subjects mentioned in the article. The corresponding author is in possession of this document.

\section{References}

1. Zarei A, Alborzi S, Dadras N, Azadi G. The effects of endometrial injury on intrauterine insemination outcome: a randomized clinical trial. Iran J Reprod Med. 2014;12:649-52.

2. Guven S, Kart C, Unsal MA, Yildirim O, Odaci E, Yulug E. Endometrial injury may increase the clinical pregnancy rate in normoresponders undergoing long agonist protocol ICSI cycles with single embryo transfer. Eur J Obstet Gynecol Reprod Biol. 2014;173:58-62.

3. Farquhar CM, Liu E, Armstrong S, Arroll N, Lensen S, Brown J. Intrauterine insemination with ovarian stimulation versus expectant management for unexplained infertility (TUI): a pragmatic, open-label, randomised, controlled, two-centre trial. Lancet. 2018:391:441-50.

4. Ashrafi M, Tehraninejad ES, Haghiri M, Masomi M, Sadatmahalleh SJ, Arabipoor A. The effect of endometrial scratch injury on pregnancy outcome in women with previous intrauterine insemination failure: a randomized clinical trial. J Obstet Gynaecol Res. 2017;43:1421-7. 
5. Potdar N, Gelbaya T, Nardo LG. Endometrial injury to overcome recurrent embryo implantation failure: a systematic review and meta-analysis. $\mathrm{Re}$ prod Biomed Online. 2012;25:561-71

6. Achache $\mathrm{H}$, Revel A. Endometrial receptivity markers, the journey to successful embryo implantation. Hum Reprod Update. 2006;12:731-46.

7. Karimzadeh MA, Ayazi Rozbahani M, Tabibnejad N. Endometrial loca injury improves the pregnancy rate among recurrent implantation failure patients undergoing in vitro fertilisation/intra cytoplasmic sperm injection: a randomised clinical trial. Aust N Z J Obstet Gynaecol. 2009;49:677-80.

8. Goel T, Mahey R, Bhatla N, Kalaivani M, Pant S, Kriplani A. Pregnancy after endometrial scratching in infertile couples undergoing ovulation induction and intrauterine insemination cycles-a randomized controlled trial. J Assist Reprod Genet. 2017;34:1051-8.
9. Gnainsky Y, Granot I, Aldo PB, Barash A, Or Y, Schechtman E, et al. Local injury of the endometrium induces an inflammatory response that promotes successful implantation. Fertil Steril. 2010;94:2030-6.

10. Barash A, Dekel N, Fieldust S, Segal I, Schechtman E, Granot I. Local injury to the endometrium doubles the incidence of successful pregnancies in patients undergoing in vitro fertilization. Fertil Steril. 2003;79:1317-22.

11. Zhou L, Li R, Wang R, Huang HX, Zhong K. Local injury to the endometrium in controlled ovarian hyperstimulation cycles improves implantation rates. Fertil Steril. 2008;89:1166-76.

12. Guven S, Kart C, Unsal MA, Yildirim O, Odaci E, Yulug E. Endometrial injury may increase the clinical pregnancy rate in normoresponders underwent long agonist protocol intracytoplasmic sperm injection cycles with single embryo transfer. Fertil Steril. 2011;6:277. 